\title{
Charged Particle Production in DIS at $\mathrm{H} 1$
}

\author{
Daniel Traynor \\ Queen Mary, University of London - Dept of Physics \\ Mile End Road, London - UK
}

\begin{abstract}
The average charged track multiplicity and the normalised distribution of the scaled momentum, $x_{p}$, of charged final state hadrons are measured in deep-inelastic $e p$ scattering at high $Q^{2}$ in the Breit frame of reference. The analysis covers the range of photon virtuality $100<Q^{2}<20000 \mathrm{GeV}^{2}$. Compared with previous results presented by $\mathrm{H} 1$ this analysis has a significantly higher statistical precision and extends the phase space to higher $Q^{2}$ and to the full range of $x_{p}$. The results are compared with $e^{+} e^{-}$annihilation data and with various calculations based on perturbative QCD using different models of the hadronisation process.
\end{abstract}

\section{Introduction}

In a recent $\mathrm{H} 1$ paper [1] the process of parton fragmentation and hadronisation is studied using the inclusive charged particle spectrum in the current region of the Breit frame of reference in Deep Inelastic Scattering (DIS) data. In this region a comparison with one hemisphere of an $e^{+} e^{-}$annihilation, taking $\sqrt{S_{e e}}=E^{*}=Q$, is possible, directly testing quark fragmentation universality.

In the Breit frame the average charged particle multiplicity, $\langle n\rangle$, is defined as the average number of charged particles in the current region per event. The scaled momentum variable $x_{p}$ is defined to be $2 p_{h} / Q$ where $p_{h}$ is the momentum of a charged track in the Breit frame. In $e^{+} e^{-}$annihilation events the equivalent variable is $2 p_{h} / E^{*}$. In this analysis the use of much higher statistics now available at high $Q$ compared to previous studies [2], and an improved understanding of the $\mathrm{H} 1$ detector and associated systematic errors, provide a much improved measurement of the scaled momenta spectra. Results are now available to $\left\langle Q>\sim 100 \mathrm{GeV}\right.$, close to the LEP1 centre of mass energy, and to the full range of $x_{p}$ (nominally, $0<x_{p}<1$ ).

\section{Data Selection}

Events are selected if the scattered positron is detected in the acceptance of the LAr calorimeter $\left(10<\theta_{e}<150^{\circ}\right)$ with an energy greater than $11 \mathrm{GeV}$. The kinematic phase space is required to be in the range $100<Q^{2}<20,000 \mathrm{GeV}^{2}$ and $0.05<y<0.6$, where $y$ is the inelasticity. The polar scattering angle for a massless parton, calculated from the positron in the QPM approximation, is required to be in the range $30<\theta_{q}<150^{\circ}$. Only primary vertex fitted tracks from the central jet chamber are used in this analysis. Good acceptance is assured by requiring tracks to be within $20^{\circ}$ to $160^{\circ}$ and transverse momenta to be above $120 \mathrm{MeV}$. In addition a variety of quality cuts are applied to remove badly measured tracks in a clean and well-modelled manner. Data are corrected for detector acceptance and resolution effects 

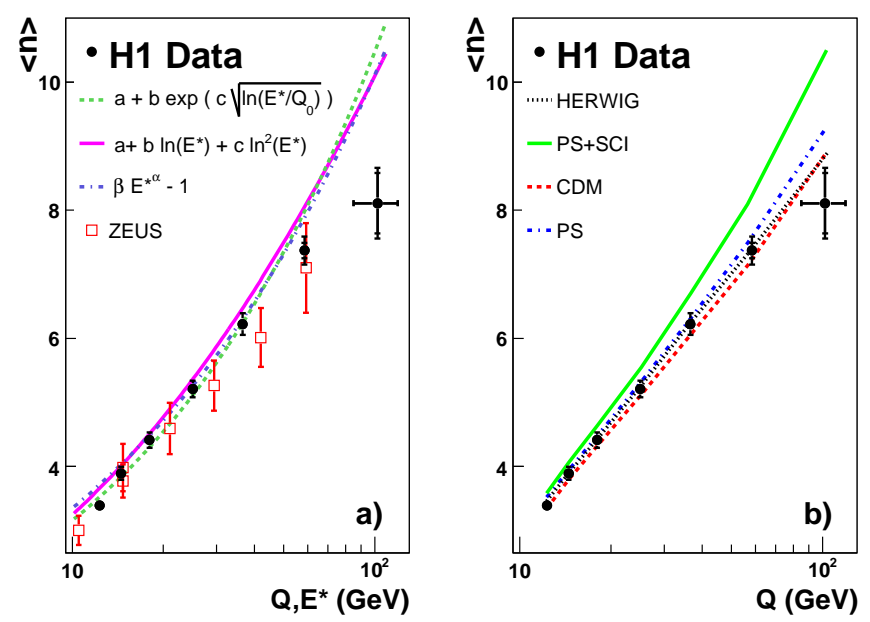

Figure 1: The average charged multiplicity as a function of $Q$. For each measurement the statistical error is shown by the inner error bar while the outer error bar represents the statistical and systematic errors added in quadrature. In addition there is a further correlated error of $2 \%$ coming from the electron energy scale uncertainty (not shown). The data are displayed at the average value of $Q$, the horizontal error bars represent the statistical errors. The data are compared with: a) parameterisations of data from $e^{+} e^{-}$experiments (taking $Q=E^{*}$ ) and with results reported by the ZEUS experiment, and b) predictions from different models of the hadronisation and parton cascade processes implemented in leading order matrix element Monte Carlo programs.

\section{Multiplicity}

In figure $1 \mathrm{a}$ ) the measurements of $\langle n>$ are compared with different parameterisations of the average charged track multiplicity per event seen in $e^{+} e^{-}$annihilation and with results from the ZEUS experiment [3]. The ZEUS results are in agreement, within the errors, with the H1 data. For most of the $Q$ range the $\mathrm{H} 1$ data are in good agreement with the parameterisation of the $e^{+} e^{-}$data. In the lowest $Q$ interval the data are slightly below the parameterisation of the $e^{+} e^{-}$data. In the highest two $Q$ intervals the measurements are clearly below the $e^{+} e^{-}$parameterisation.

In figure 1b) a comparison is made with different models of the hadronisation and parton cascade processes implemented in leading order matrix element Monte Carlo programs. The models show good agreement with our data except for the one with soft colour interactions which significantly overestimates the multiplicity.

\section{Scaled Momentum}

A summary of the results for the scaled momentum spectra is presented in figure $2 \mathrm{a}$ ) as a function of $x_{p}$ for different $Q$ intervals, where each $Q$ interval has been scaled by an additional factor ten for improved visual display, and are compared with the parton shower 

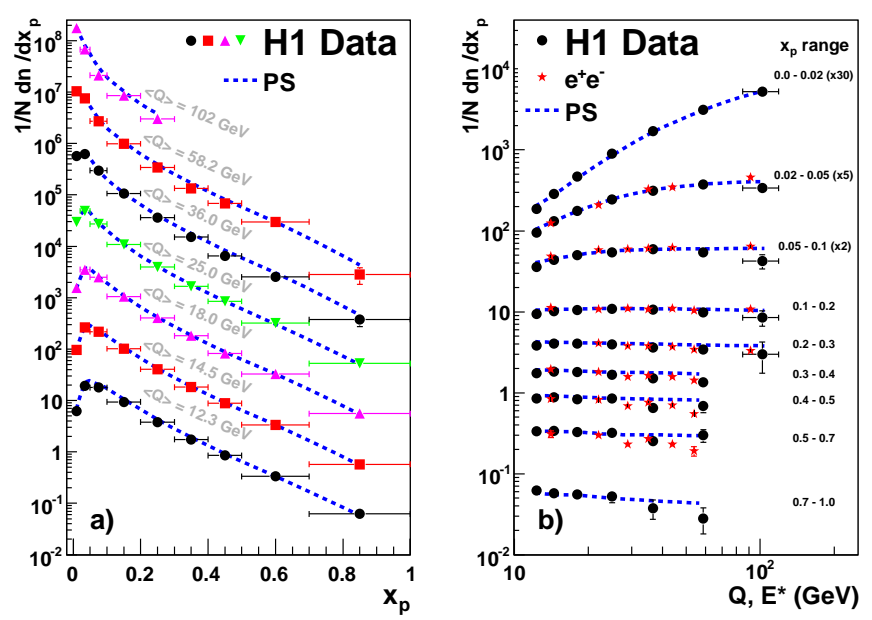

Figure 2: The measured normalised distributions of the scaled momentum, $\frac{1}{N} d n / d x_{p}$, as a function of $Q$ for nine different $x_{p}$ regions. In addition there is a further correlated error of $\sim 0.5-7 \%$ (increasing with $x_{p}$ ) coming from the electron energy scale uncertainty (not shown). The data are compared to predictions from different Monte Carlo programs and with results from $e^{+} e^{-}$annihilation experiments. See figure 1 for more details.

(PS) Monte Carlo prediction. In figure 2b) the results are presented as a function of $Q$ for different $x_{p}$ intervals and are compared with the $e^{+} e^{-}$annihilation data and the PS Monte Carlo prediction. More detailed studies were shown in [4].

The results broadly support the concept of quark fragmentation universality in $e p$ collisions and $e^{+} e^{-}$annihilation. A small multiplicity depletion compared to $e^{+} e^{-}$is observed at low $Q$ which can be attributed to higher order QCD processes occurring as part of the hard interaction in $e p$ scattering but not in $e^{+} e^{-}$annihilation. At high $Q$ a large depletion is observed.

More detail studies show that both the colour dipole model (CDM) and the PS model provide an acceptable description of the data, with the CDM model predicting a slightly harder spectrum. Both models tend to overestimate the multiplicity at higher $Q$. The SCI model predicts a much softer spectrum than the other two models and is disfavoured by the data. This could be due to the additional gluon interactions in the SCI model which soften the spectra of partons produced by the parton shower. The data have a preference for predictions where the Lund string model of hadronisation is used (PS and CDM). HERWIG (cluster hadronisation) predicts a spectrum that is too hard compared to the data. At high $x_{p}$ HERWIG even fails to reproduce the observed scaling violation and predicts a flat spectrum in $Q$.

In figure 3 the data are compared with the predictions obtained from the NLO calculation for three different parameterisations of the fragmentation function in an infrared safe region. The uncertainties associated with the change in scale or the protons parton destiny function (PDF) are significantly smaller than the differences between these parameterisations. The different fragmentation function parameterisations give different results but it is evident that 
none of them can describe the scaling violations seen in the data.
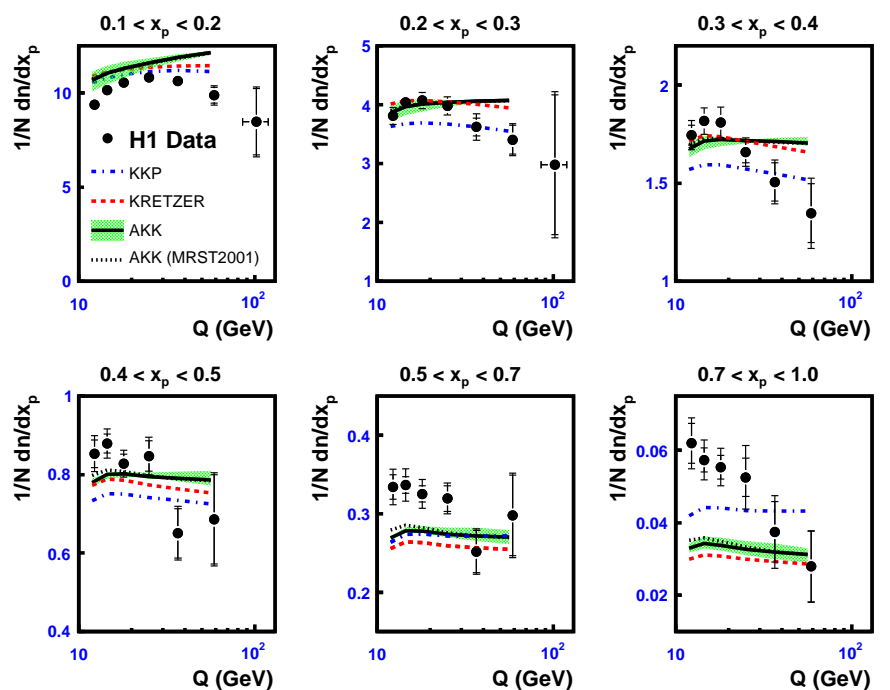

Figure 3: The measured normalised distributions of the scaled momentum, $\frac{1}{N} d n / d x_{p}$, as a function of $Q$ for six different $x_{p}$ regions where there exist infra red safe NLO QCD predictions. The data are compared to NLO QCD CYCLOPS predictions for $Q<60 \mathrm{GeV}$ using three different fragmentation functions: KKP (dot-dashed line), AKK (solid), and KRETZER (dashed). The typical scale uncertainties for the AKK predictions are shown as a shaded band. As standard the CTEQ6.1 PDF is used, the effect of using the MRST2001 PDF for the AKK predictions are also shown (dotted). See figure 2 for more details.

\section{Conclusion}

The results broadly support the concept of quark fragmentation universality in $e p$ collisions and $e^{+} e^{-}$annihilation. The Monte Carlo which model the parton cascade by implementing either the colour dipole model or parton showers describe the data. The string model of hadronisation provides a better description of the data than the cluster model as implemented in HERWIG. The NLO predictions fail to describe the scaling violations seen in data.

\section{References}

[1] F. D. Aaron et al. [H1 Collaboration], Phys. Lett. B 654 (2007) 148.

[2] S. Aid et al. [H1 Collaboration], Nucl. Phys. B 445 (1995) 3; C. Adloff et al. [H1 Collaboration], Nucl. Phys. B 504 (1997) 3.

[3] S. Chekanov et al. [ZEUS Collaboration], JHEP 0806 (2008) 061

[4] Slides:

http://indico. cern. ch/contributionDisplay. py? contribId=194\&sessionId=13\&conf Id=24657 